\title{
Transferencia de conocimientos: Caso Apovinces
}

\section{Transfer of knowledge: Case Apovinces}

\author{
Econ. Galo Mauricio Durán Salazar \\ Teresa Meza Clark \\ Jorge Meza Clark \\ Universidad de Guayaquil, Ecuador
}

Autor para correspondencia: galo.duransa@ug.edu.ec, teresa.mezacl@ug.edu.ec, jorge.mezacl@ug.edu.ec

Fecha de recepción: 16 de Enero de 2017 - Fecha de aceptación: 25 de Marzo de 2017

Resumen: La presente investigación realiza un estudio sobre transferencia de conocimientos en la Asociación de productores de cacao Apovinces en Ecuador, organización que ha crecido de manera importante, generando asociatividad en los agricultores, para ello ha capacitado a sus socios y agricultores en todos los temas que favorezcan la producción de cacao; sus integrantes entienden la importancia de trabajar responsablemente el producto, dado que se exporta hacia Europa, mercado que es exigente en calidad. Apovinces y la Universidad de Guayaquil tienen un vínculo para desarrollar la productividad, esto es a través de la Facultad de Ciencias para el Desarrollo, se envían estudiantes a las haciendas para enseñar a trabajar la siembre y cosecha a los agricultores, de manera que obtengan independencia financiera y no migren hacia la ciudad. De acuerdo a la literatura revisada el proceso de Apovinces responde al modelo de Transferencia de tecnología Triple Hélice. Se concluye que se debe difundir este caso exitoso para otros sectores agricultores del país, de manera que los productores puedan exportar sus productos directamente, evitando la cadena de intermediarios.

Palabras claves: transferencia de conocimientos; transferencia de tecnología; modelo triple hélice; caso de estudio agrícola; apovinces

Abstract: The research carries out a study on transfer of knowledge in the Association of Cocoa
Producers Apovinces in Ecuador, an organization that has grown significantly, generating
associativity in farmers, it has trained its partners and farmers on all issues that favor Cocoa
production; Its members understand the importance of working responsibly the product, it is
exported to Europe, a market that is demanding in quality. Apovinces and the University of
Guayaquil have a link to develop productivity through the Faculty of Sciences for Development,
students are sent to the haciendas to teach to work the sow and harvest to the farmers, so that they
obtain independence and do not migrate to the city. According to the literature reviewed, the
Apovinces process responds to the Triple Helix Technology Transfer model. It is concluded that
this successfully case should be showed to other agricultural sectors of the country, so that farmers
can export their products directly, without the chain of middle man.

Key Words: transfer of knowledge; technology transfer; triple propeller model; agricultural case study; apovinces 


\section{Introducción}

El Aprendizaje es un proceso muy complejo que ha originado diversas teorías, una definición de acuerdo Moreno (2012) "El aprendizaje es relativamente perdurable en la percepción de las personas en su interacción con el medio y sus experiencias de vida o en la capacidad de comportarse de una determinada manera, la cual resulta de la práctica o de alguna otra forma de experiencia". De esta forma el aprendizaje, es el inicio de la Gestión de Conocimientos (GC), este tema GC se remonta a pensadores como Smith (1776) quien describió Pin-making como la especialización que promueve el aprendizaje basado en la experiencia; Marshall (1920) argumentó que en aglomeraciones regionales, las ideas son fácilmente tomadas y discutidas; Weber (1978) analizó la capacidad de las burocracias de los entes para aprender de sus experiencias y aportar en las gestiones propias. Luego la Escuela de Carnegie mejorada por Cyert and March (1963) transformó todas la observaciones en una teoría denominada "el aprendizaje organizacional y la gestión del conocimiento”.

La investigación sobre la Transferencia de Conocimientos (TC) implica disciplinas como la economía, sistemas de información, comportamiento organizacional, lo que ha contribuido a la integración de estas. Según Jasimuddin y Zhang (2009) en la nueva economía el conocimiento organizacional se reconoce como la principal fuente de ventaja competitiva de la organización y en consecuencia, su transferencia es explicita como un asunto estratégico; Argote, McEvily, \& Reagans (2003) indica que la gestión del conocimiento basado en la revisión de la literatura se genera por Creación, Retención y Transferencia de Conocimientos; la creación de conocimiento implica un nuevo conocimiento o habilidad en una organización; la retención del conocimiento se refiere a la utilización de la nueva habilidad a través del tiempo, es decir, la persistencia en su aplicación; y la transferencia de conocimiento se aplica cuando la experiencia adquirida en una unidad afecta a otra.

A nivel de compañías Blome, Schoenherr, \& Eckstein, (2013) dentro de las empresas, el conocimiento es un bloqueo fundamental de la construcción para las organizaciones, ya que permite a las empresas enfrentar desafíos contemporáneos manejar complejidades y mantenerse competitivas. También puede definirse a la transferencia de conocimientos como al proceso mediante el cual un ente se afecta por la experiencia de otro. Según Barragán (2009):

"La gestión del conocimiento como la habilidad individual o colectiva para generar, difundir, compartir y utilizar tanto el conocimiento tácito como explícito a partir de la asimilación de la información que se transfiere en forma de conocimiento y se transforma en experiencia de organizaciones o individuos; convirtiéndose así en una herramienta de aprendizaje útil que permite la aplicación del conocimiento para aportar valor dentro de una organización, economía o sociedad” (p.5). Básicamente la Transferencia de Conocimientos, se genera por experiencia adquirida en el día, el cual puede replicarse a otras organizaciones facilitando el aprendizaje.

Según Szulanski (1996), el proceso del conocimiento de transferencia dentro de una empresa podría dividirse en cuatro etapas: Iniciación, implementación, ramp-up (aumento) e integración. La primera etapa se caracteriza por todos los eventos que conducen a la decisión de transferir. 
Una vez tomada la decisión, la etapa de implementación comienza con la resolución para continuar en el proceso de transferencia de conocimiento. Cuando el destinatario comienza utilizando el conocimiento transferido, comienza la etapa de ascenso, es decir, después del primer momento de uso. En esta etapa todos los participantes tienen la oportunidad de resolver problemas que podrían presentarse, y con ello acumulan más conocimientos y aumentan su aprendizaje real. La etapa de integración se realiza una vez el conocimiento transferido se convierte en rutinas, y por lo tanto institucionalizado. También creemos que después de la integración mientras que los beneficiarios logran resultados exitosos, período de inercia breve, después de lo cual los receptores comenzar nuevamente el proceso de transferencia de conocimiento.

A nivel de Tecnológico, la Transferencia de Tecnología TT, se define como la transferencia de una fuente a otra de cualquier técnica, producto o proceso, equipamiento físico o método de elaboración (Schon, 2011). El término transferencia de tecnología se refiere a una serie de estrategias que promuevan la transferencia de nuevas innovaciones, conocimientos, tecnologías, prácticas y / o habilidades de un entorno a otro, es decir, de la investigación a la práctica. (Barcker, 2002). La TT y la difusión de innovaciones son diferentes, aunque se superponen (Roget, 2009).

De acuerdo a Rogers (2010) la TT está orientada al productor (por ejemplo, el innovador crea estrategias para fomentar el uso de la innovación), mientras que la difusión es más amplia e incluye la propagación planificada y espontánea de las innovaciones. Backer (1991) definió la transferencia de tecnología como proceso de transmisión de información para obtener la adopción solicitud. Según Tenkasi y Mohrman (1995) ampliaron la definición de transferencia de tecnología para incluir la transmisión no sólo de información sino también de ideas, conocimientos y equipo. Brown en el (2000) sugirió que la transferencia de tecnología es más que la transmisión de información y que en realidad es un proceso de cambio de comportamiento.

\section{Modelos de Transferencia de conocimientos}

Los modelos de transferencia de conocimientos (Mcadam \& Mccreedy, 1999) se pueden clasificar en:

- Modelos categóricos del conocimiento: su esencia es básicamente teórica.

- Modelos de capital intelectual: puede presentarse en dos categorías: el capital humano y el estructural / organizacional.

- Modelos socialmente construidos: están relacionados a los procesos sociales y el aprendizaje organizacional.

- Otra forma de clasificar los modelos de transferencia de conocimientos Rodríguez (2006):

- Almacenamiento, acceso y transferencia de conocimientos: se basa en la obtención de información y datos.

- Sociocultural: basados en la cultura de las personas promoviendo cambios de actitud, confianza, creatividad generando la importancia del valor del conocimiento en los miembros de una organización, creando así colaboración y comunicación. 
- Tecnológicos: Basados en el uso del sistema informático así como herramientas tecnológicas.

\section{Modelo de Transferencia de Tecnología}

Se puede encontrar tres modelos de Transferencia de Tecnología, el Lineal, Dinámico, y Triple Hélice. El modelo Lineal según Siegel, Waldman, \& Atwater (2004) se refiere a los descubrimientos científicos que se generan y que deben patentarse teniendo como actores a la universidad-centro de investigación-oficina de transferencia de resultados (OTRI) -y las empresas, el proceso de lo puede observar en la figura 1, básicamente se busca patentar y explotar el descubrimiento realizado.

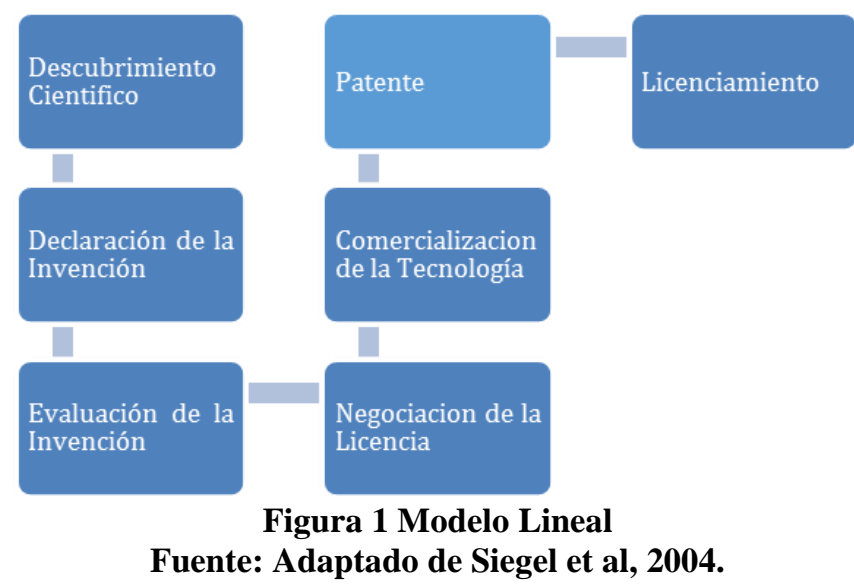

El modelo dinámico, que es una mejora del lineal, tiene como objetivo la transferencia del conocimiento a través de la comercialización o difusión utilizando variables que son críticas para este proceso:

- El entendimiento intercultural.

- Las habilidades de la negociación.

- Los incentivos para la negociación.

El modelo Triple Hélice que de acuerdo a Etzkowitz \& Leydesdorff (2000) capta de manera relevante las interacciones entre universidad-industria-gobierno, de manera que la universidad contribuya al desarrollo económico y social a nivel nacional a través de organizaciones hibridas como centros tecnológicos, parques científicos e incubadoras, este modelo se presenta en la figura 2

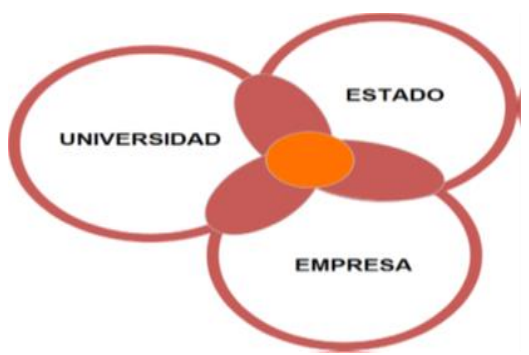

Figura 2 Modelo Triple Hélice

Fuente Adaptada de Etzkowitz y Leydesdorff (2000) 


\section{Asociación de productores orgánicos de Vinces APOVINCES}

Se crea en el año 2006, en el cantón Vinces, perteneciente a la provincia de Los Ríos, por parte de un grupo pequeño de cultivadores, quienes tenían como objetivo mejorar e impulsar la agricultura limpia a través de asociaciones de campesinos, que en trabajo conjunto puedan mejorar la producción y productividad de los socios, afectando positivamente al entorno de los campesinos, información sobre la organización se puede encontrar en http://apovinces.org/index.php.

Ubicada en una zona costera con particular riqueza hídrica, los campesinos se especializan en el cultivo de cacao, además de maíz, arroz soya y frutales, con formado por 300 a 400 agricultores, quienes poseen en promedio 8 hectáreas de terreno para cultivo de cacao nacional y su exportación. (Ramos, 2014, pág. 5)

De acuerdo al censo de población y vivienda (INEC, 2010) Vinces tiene una población de 71.736 habitantes, generando el sector del cacao aproximadamente 41.000 empleos frente a 39.008 el sector bananero, lo cual indica que el cantón Vinces depende de los bienes agrícolas. Antes de la creación de la asociación Apovinces, los agricultores de cacao negociaban su producto con intermediarios a precios bajos, llevándose ellos el mayor margen de ganancia, dado que ese es el problema principal de los productores agrícolas, la falta de visión de negocios, es decir, no hacer la venta directa (Durán, Salazar, \& Meza, 2016, pág. 381).

Con la creación de la Asociación Apovinces, los productores del sector se han ido beneficiando, en vista que la organización ha crecido de manera importante en el manejo administrativo del negocio y en la exportación de cacao con calidad. El proceso aplicado en la asociación como transferencia de conocimiento basado en la experiencia se puede resumir de la siguiente manera:

- Comercialización asociativa: consiste en crear asociaciones sin fines de lucro, donde el objetivo es que los beneficios que genere el sector se retribuyan en la comunidad, en vista que la asociación vende por volumen directamente a empresas exportadoras o a mercados internacionales, evitando al intermediario en la cadena de comercialización.

- Capacitación a los productores de cacao: consiste en agricultura limpia, de disminuir los impactos ambientales, tratando de realizar de mejorar podas, almacenamiento, secado y comercialización.

- Exportar directamente considerando las certificaciones que posee la organización como son CERES de tipo orgánica, RAIN FOREST agroecológica y de comercio JUSTO FLO. Esos tres sellos le permite a APONVINCES vender su cacao a un precio de los más altos del país, permitiéndole competir incluso con empresa transnacionales, lo anterior se da por el trabajo minucioso de cuidado orgánico y agroecológico.

- Vínculo con la Universidad de Guayaquil, lo que le permite generar un respaldo de calidad en la producción de cacao, base para la obtención de las tres certificaciones mencionadas; a través de la Facultad de Ciencias para el Desarrollo quien realiza pruebas de semillas de mejoramiento en las haciendas de los agricultores.

- Evitar la migración de los jóvenes vinceños a grandes ciudades, ayudándolos a trabajar sus tierras para que puedan generar riquezas. 
Lo anterior ha permitido que desde el año 2012, APOVINCES exporte directamente a empresas suizas atraídas por las certificaciones y el respaldo de la Universidad de Guayaquil. Los agricultores entregan su producto a la asociación y esta les cancela a determinado valor; además las empresas certificadoras entregan un premio para APOVINCES, que luego lo distribuye asociativamente a sus socios.

Según (Castro \& Meza, 2015) organizaciones como APOVINCES, pueden mejorar su economía de la siguiente forma:

- Aplicar Modelos Transferencia de tecnología.

- Mejorar la infraestructura para acopio de producto.

- Fortalecer el proceso de asociación de productores.

- Mejorar los controles de calidad del producto.

- Reducir los costos de la cadena.

\section{Discusión}

Se puede indicar que APOVINCES, su gestión en cuanto a Transferencia de conocimiento, ha sido exitosa, le permite exportar y recibir premios por el producto de calidad, basado en certificaciones que acreditan los procesos del cacao, las capacitaciones que han recibido los agricultores y la forma en la que se ha tratado de involucrarlos, de tal manera que tengan la necesidad de utilizar productos de calidad para producir, además de hacer los procesos adecuados para fertilizar y cosechar el producto. En cuanto a un modelo de Tecnología responde al modelo teórico Triple Hélice, en vista que interviene la Universidad, la asociación Apovinces y el Estado.

La universidad participa facilitando los estudios para mejorar los procesos de producción del cacao en las haciendas de los agricultores; la asociación Apovinces realizando prácticas adecuadas como empresa, generando beneficios a sus socios, además del reconocimiento internacional por vender un producto de calidad; el Estado por las leyes que se realizan como por ejemplo la Asociatividad, que busca que el beneficio lo genere el productor, reduciendo el número de intermediarios para el sector.

\section{Conclusiones}

La transferencia de conocimientos es importante permite el crecimiento y sobrevivencia de una organización, para ello, se debe aprender de los errores, y mejorar continuamente, a través de la experiencia que se adquiere con el tiempo. Apovinces es una institución sin fines de lucro, creada por agricultores, bajo la figura se asociatividad, conforme lo establecen las leyes ecuatorianas; ha tenido un crecimiento importante como empresa, produce cacao de calidad, en base a certificaciones internacionales lo cual permite que sea atractivo su cacao, por ello recibe premios en sus exportaciones; los agricultores han sido capacitados de tal manera que conocen la importancia que tienen los procesos de sembrado y cosecha del producto.

Este modelo, debe ser replicado a otros productores, a fin de que conozcan que trabajar bajo una asociación beneficia a todos los agricultores y permite independencia financiera y que 
por ejemplo no se realicen procesos migratorios de las personas a las grandes ciudades, donde encontrar un trabajo fijo, no es fácil. Para futuras investigaciones, este modelo podría utilizarse dentro del Modelo Macro de Transferencia de Tecnología para el Ecuador 2013, elaborado por Secretaria Nacional de Planificación y Desarrollo SENPLADES.

\section{Bibliografía}

Argote, L., McEvily, B., \& Reagans, R. (2003). Managing Knowledge in Organizations: An Integrative Framework and Review of Emerging Themes. Management Science, 49(4), 572-574.

Barcker, T. (2002). Drug abuse technology transfer. Journal of Substance Abuse Treatment, 22(4), 245-257. doi:http://dx.doi.org/10.1016/S0740-5472 (02)00228-3

Barragán, A. (2009). Aproximación a una taxonomía de modelos de gestión del conocimiento. Intangible Capital, 65-101. doi:10.3926/ic.2009.v5n1.p65-101

Blome, C., Schoenherr, T., \& Eckstein, D. $\quad$ (2013). The impactofknowledgetransferandcomplexityonsupplychain flexibility: A knowledge-basedview. Int. J.ProductionEconomics, 1-9.

Brown, B. S. (2000). From research to practice: The bridge is out and the water is rising. Advances in Medical Sociology, 7, 345-365.

Castro, P., \& Meza, T. (2015). Ecuador: educación en tecnologías de agro negocios por medio del modelo laboratorio vivo en cacao fino de aroma en el cambio de la matriz productiva. Revista Observatorio de la Economía Latinoamericana, Ecuador. Obtenido de http://www.eumed.net/cursecon/ecolat/ec/2015/laboratorio-vivo.html

Cyert, R., \& March, J. (1963). A Behavioral Theory of the Firm. Englewood Cliffs: Prentice-Hall.

Durán, G., Salazar, V., \& Meza, T. (2016). Estrategias de Trazabilidad para la exportación de cacao. Revista Publicando, 3(8), 381. Obtenido de http://www.rmlconsultores.com/revista/index.php/crv/issue/view/VOL.\%203\%20No.\%208

Etzkowitz, H., \& Leydesdorff, L. (2000). The dynamics of innovation: from National Systems and «Mode 2» to a Triple Helix of university-industry-government relations. Research Policy (29), 109-123.

INEC. (2010). Censo de población y vivienda.

Instituto Nacional de Propiedad Industrial. (s.f.). Obtenido de Instituto Nacional de Propiedad Industrial: http://www.inapi.cl/portal/orientacion/602/w3-article-693.html

Jasimuddin, S., \& Zhang, Z. (2009). The symbiosis mechanism for effective knowledge transfer. Journal of Operational Research Society, 60(5), 706-716.

Marshall, A. (1920). Principles of Economics: An Introductory Volume. London. 
Mcadam, R., \& Mccreedy, S. (1999). A critical review of knowledge management. The Learning Organization, 6(3), 91-100.

Moreno, S. (2012). Los aprendizajes significativos y la educación. Enfoque Humanístico (21), 1-21. Obtenido de http://media.wix.com/ugd/b33ce8_5278bdb703f5a6603879abc58f34aec6.pdf

Ramos, M. (2014). Autogestión y fortalecimiento asociativo de base familiar campesina en la producción de cacao. Vinces.

Rodríguez, D. (2006). Modelos para la creación y gestión del conocimiento: Una aproximación teórica. Educar (37), 25-59.

Rogers, E. (2010). A Prospective and Retrospective Look at the Diffusion Model. Journal Of Health Communication, 9(1), 13-19. doi:http://dx.doi.org/10.1080/10810730490271449

Roget, N. (2009). Evidence-based prevention and treatment, dissemination and adoption of. Encyclopedia of substance abuse prevention, treatment, \& recovery, 379-382.

Schon. (2011). Research to practice in addiction treatment: Key terms and a field-driven model of technology transfer. Journal of Substance Abuse Treatment, 41(2), 169-178. doi:http://dx.doi.org/10.1016/j.jsat.2011.02.006

Siegel, D., Waldman, D., \& Atwater, L. (2004). Toward a model of the effective transfer of scientific knowledge from academicians to practitioners: Qualitative evidence from the commercialization of university technologies. Journal of Engineering and Technology Management, 21(1), 115-142.

Smith, A. (1776). An Inquiry into the Nature and Causes of the Wealth of Nations.

Szulanski, G. (1996). Exploring internal stickiness: impediments to the transfer of best practice within the firm. Strategic Management Journal (17), 27-43. Obtenido de http://www.sciencedirect.com/science/article/pii/S1132055914000040

Tenkasi, R. V., \& Mohrman. (1995). Technology transfer as collaborative learning. Reviewing the Behavioral Science Knowledge Base on Technology Transfer. National Institute on Drug Abuse Monograph, 155, 147-169.

Universidad Autónoma de Barcelona. (s.f.). Obtenido de Universidad Autónoma de Barcelona: http://www.uab.cat/web/investigar/itinerarios/innovacion-transferencia-yempresa/transferencia-del-conocimiento-1345667266489.html

Webber. (1978). Economy and Society: An Outline of Interpretive. Berkeley. 